\title{
Propriétés diélectriques du nitrure d'aluminium compacté, fritté et dopé au chrome
}

\author{
A. Kumar (*), J. P. Lecompte (**) et A. Moliton (*) \\ U.E.R. des Sciences, 123, avenue Albert Thomas, 87060 Limoges Cedex, France
}

(Reçu le ler juillet 1981, révisé le 18 janvier 1982, accepté le 20 janvier 1982)

\begin{abstract}
Résumé. - L'étude diélectrique de AIN fritté sous charge nous a montré que les pertes diélectriques, surtout importantes aux plus basses fréquences, proviennent de deux origines :

- l'une est due à des défauts de réseau du type « imperfection chimique » et correspond à la présence de AlON; elle se traduit par une polarisabilité interfaciale dont le terme dipolaire produit un pic d'absorption visualisé aussi par l'expérience.

- l'antre est de nature "imperfection physique " liée à des défauts de réseau dans lesquels s'accumulent des charges dont on a pu discuter la liaison avec les défauts. La conductivité de cette nature peut être accrue jusque vers $650 \mathrm{~K}$ par un " dopage " à l'aide de chrome métallique, qui augmente le nombre de charges liées aux défauts de réseau.
\end{abstract}

\begin{abstract}
The dielectric study of hot pressed AlN has shown that the dielectric losses which are particularly important at lower frequencies arise from two processes :

- one is due to lattice defects of the "chemical imperfection " type and corresponds to the presence of AlON and which is characterized by an interfacial polarizability in which the dipolar term results in the observed absorption peak ;

- the other is a " physical imperfection " connected with lattice defects in which the charges are accumulated. The nature of the relation between the charge distribution and the defects is discussed. The conductivity resulting from this process can be increased, up to about $650 \mathrm{~K}$, by " doping " with metallic chromium, which increases the number of charges attached to lattice defects.
\end{abstract}

1. Introduction. - Le nitrure d'aluminium fritté est bien connu comme matériau céramique résistant à haute température [1, 2], ce qui permet d'envisager sa mise en ouvre dans des applications faisant appel traditionnellement à des métaux (turbomachines, moteurs à haut rendement). Dans ce contexte, l'étude des propriétés diélectriques paraît importante afin de définir le comportement isolant à haute température du nitrure d'aluminium, en fonction de la température, de l'influence des ajouts et des dopants.

Pour comprendre la nature et l'origine des pertes observées dans ces matériaux, l'étude de la variation de la permittivité diélectrique avec la température et la fréquence est nécessaire.

Des travaux [3] ont indiqué jusqu'à $700 \mathrm{~K}$ les variations de la permittivité diélectrique mais aucune

(*) Laboratoire de Physique Moléculaire.

(**) Laboratoire de Céramiques Nouvelles. CNRS, L.A. 320. explication des phénomènes n'a pu être fournie. Une autre publication [4] ayant donné les caractéristiques diélectriques à température ambiante, il paraissait intéressant de poursuivre ces études à basse et haute température vu le peu d'informations que nous avions sur ce matériau.

2. Méthodes expérimentales. - 2.1 MÉTHOdE D'OBTENTION DES ÉCHANTILLONS ET CARACTÉRISTIQUES PHYSICO-CHIMIQUES DES PRODUITS ÉTUDIÉS. - Nous avons utilisé des poudres commerciales de nitrure d'aluminium $\left({ }^{1}\right)$, d'oxyde de calcium $\left({ }^{2}\right)$, et de chrome $\left({ }^{3}\right)$. La mesure de la surface spécifique de la poudre de nitrure d'aluminium nous a donné la valeur

( $\left.{ }^{1}\right)$ AlN, $99 \%$ de pureté, granulométrie $<50 \mu \mathrm{m}$, Koch Light U.K.

( $\left.{ }^{2}\right) \mathrm{CaO}, \mathrm{RP}$, granulométrie $<31 \mu \mathrm{m}$, Prolabo.

( $\left.{ }^{3}\right) \mathrm{Cr}$ métal, granulométrie $<38 \mu \mathrm{m}$, Herman C. Starck Berlin. 
de $2,7 \mathrm{~m}^{2} / \mathrm{g}$. La contamination de l'oxygène de cette poudre a été estimée par activation neutronique à $2,7 \%$ en masse, l'oxygène se trouvant sous forme d'oxynitrure d'aluminium après frittage.

Dans un premier temps, l'étude a été réalisée sur de la poudre compactée sous vide dans un moule de $3 \mathrm{~cm}$ de diamètre sous une pression de $2 \mathrm{t} / \mathrm{cm}^{2}$. L'épaisseur finale de la pastille est de $2 \mathrm{~mm}$, la porosité étant de $45 \%$. Ensuite, suivant des conditions définies lors de travaux antérieurs [1], nous avons obtenu des céramiques denses en utilisant la technique du frittage sous charge.

Différentes céramiques de nitrure d'aluminium sans ajout, avec ajout d'oxyde de calcium $(0,7 \%$ en mole) et de chrome métallique $(0,1 \%-0,5 \%-2 \%$ en mole) ont été préparées à une température de $2020 \mathrm{~K}$ sous une pression de $20 \mathrm{MPa}$ pendant $20 \mathrm{~min}$. L'étude par diffraction $\mathrm{X}$ nous a montré que l'oxynitrure d'aluminium n'était pratiquement pas présent dans ces nouveaux échantillons. Nous avons obtenu une densification totale (porosité résiduelle non mesurable) des pastilles pour une épaisseur finale de $3 \mathrm{~mm}$.

2.2 TECHNIQUE EXPÉRIMENTALE DE MESURE DES CARACTÉRISTIQUES DIÉLECTRIQUES. - Nous avons utilisé un spectromètre de mesures diélectriques automatisé [5]. Il permet des mesures de la partie réelle $\varepsilon^{\prime}$ et imaginaire $\varepsilon^{\prime \prime}$ de la permittivité relative complexe $\varepsilon^{*}=\varepsilon^{\prime}-j \varepsilon^{\prime \prime}$ dans le domaine de fréquence s'étendant de $700 \mathrm{~Hz}$ à $1 \mathrm{MHz}$; les condensateurs de mesures, plans et à anneau de garde, permettent les études diélectriques dans deux plages de températures :

- de $77 \mathrm{~K}$ à $380 \mathrm{~K}$, avec du téflon comme isolant entre les électrodes et la cage de Faraday qui sert d'enceinte au condensateur [5];

- de $300 \mathrm{~K}$ à $1000 \mathrm{~K}$ avec du nitrure de bore comme isolant, choisi à cause de sa résistance aux hautes températures, de ses propriétés mécaniques et électriques. Les connexions électriques (qui correspondent toujours à un condensateur du type trois bornes pour limiter l'effet de capacité des liaisons) sont blindées par un tuyau en acier réfractaire refroidi par une circulation d'eau (Fig. 1).

La température des échantillons est relevée à l'aide d'un thermocouple nickel chrome-nickel allié, du type thermocoax blindé et dont la gaine métallique est en contact avec l'anneau de garde ainsi relié à la masse. La conversion : d.d.p. (mesurée avec un voltmètre à sortie IEEE) fournie par le thermocouple-température, est effectuée directement par le calculateur qui pilote la manipulation diélectrique, à partir de relations analytiques du type $T=a+b V+c V^{2}$; les paramètres $a, b, c$ sont ajustés pour différentes plages de température qui correspondent chacune à un intervalle de 100 degrés (par exemple intervalles $173 \mathrm{~K}$ 273 K, 273 K-373 K, ..., 873 K-973 K...). L'erreur ainsi introduite par cette conversion est de l'ordre du degré, et correspond à une précision largement suffisante pour le type des mesures effectuées.

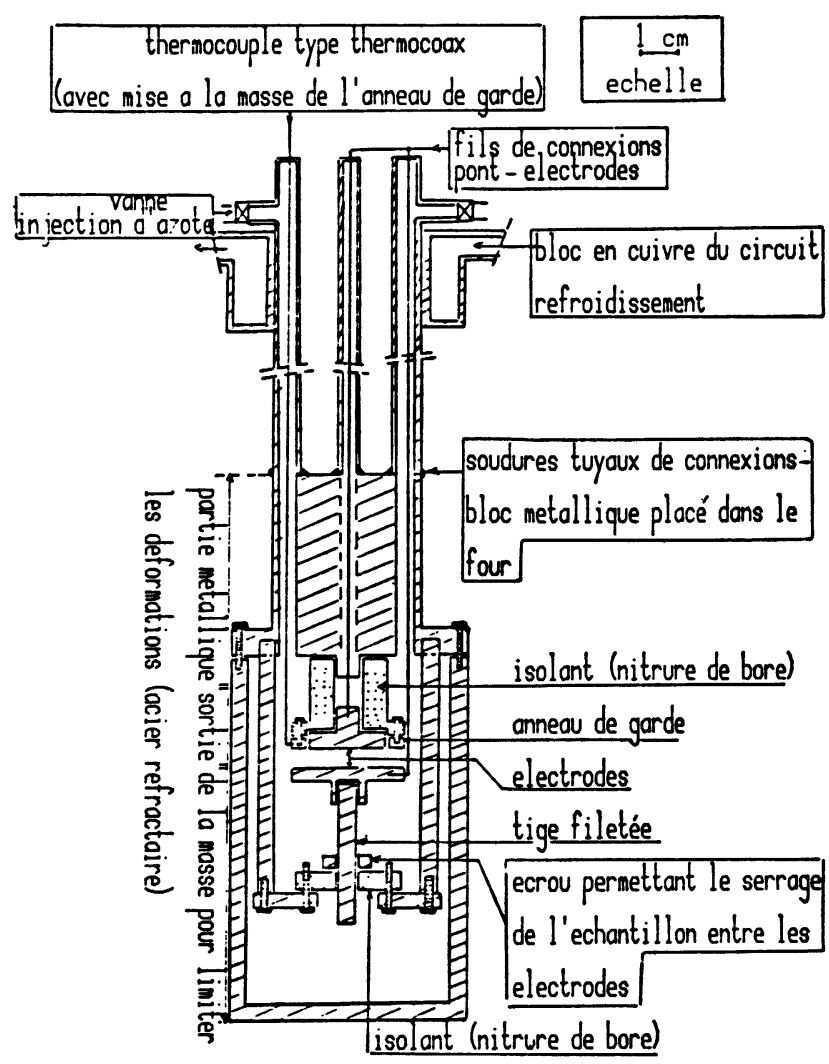

Fig. 1. - Condensateur de mesure à haute température.

[High temperature dielectric cell.]

Les faces planes des échantillons ont été rectiffées, puis polies préalablement aux mesures; d'une manière générale, nous avons renoncé au laboratoire à la métallisation des échantillons soit par évaporation sous vide (diffusion de métal qui modifie l'épaisseur des échantillons, ce phénomène étant particulièrement important dans le cas des couches minces de polymères que l'on moule directement entre les deux électrodes), soit par application d'une peinture à l'argent (dont les diluants peuvent modifier les propriétés diélectriques de surface).

Compte tenu des ordres de grandeur mesurés (assez élevés pour un diélectrique), les incertitudes relatives sont dans tous les cas inférieures à $2 \%$ pour $\varepsilon^{\prime}$ et à $3 \%$ pour $\varepsilon^{\prime \prime}$ (la précision est en réalité d'autant meilleure que la fréquence d'étude est basse). Les mesures diélectriques des échantillons ont été réalisées dans tous les cas à 8 fréquences que l'on s'est fixées : $700 \mathrm{~Hz}$, $1 \mathrm{kHz}, 3 \mathrm{kHz}, 10 \mathrm{kHz}, 30 \mathrm{kHz}, 100 \mathrm{kHz}, 300 \mathrm{kHz}$, $1 \mathrm{MHz}$.

3. Présentation des résultats diélectriques expérimentaux. - De façon générale, les résultats expérimentaux sont donnés sous la forme de courbes (tracées point par point à partir des valeurs stockées par le calculateur qui pilote la manipulation) représentant à différentes fréquences $v$ (avec $v=700 \mathrm{~Hz}$, $1 \mathrm{kHz}, 10 \mathrm{kHz}, 100 \mathrm{kHz}, 1 \mathrm{MHz}$ ) les évolutions de $\varepsilon^{\prime}$ et $\varepsilon^{\prime \prime}$ en fonction de la température $T$.

Sur la figure 2 est représenté le spectre diélectrique 


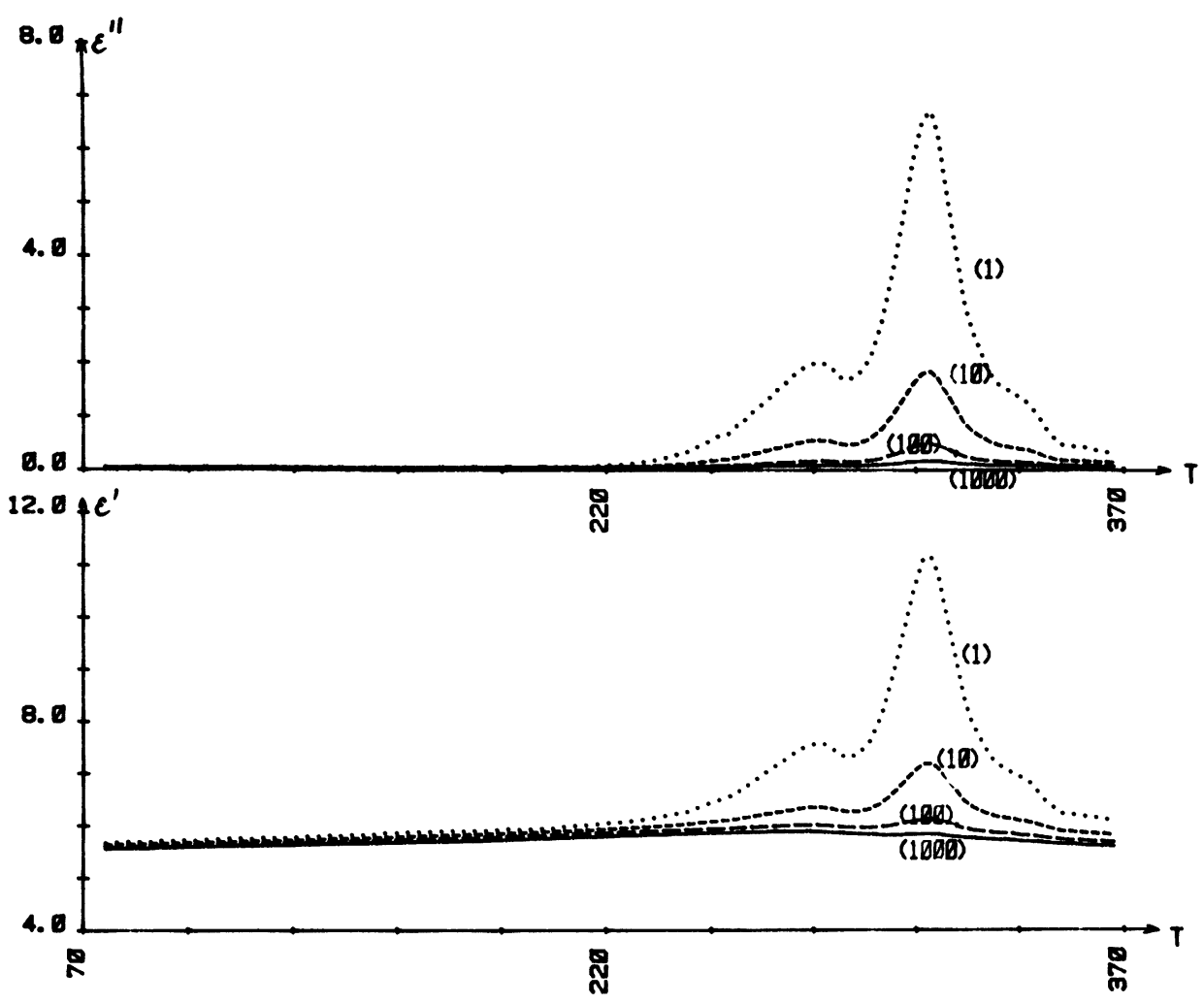

Fig. 2. - Courbes « basse température " $\varepsilon^{\prime}=f(T)$ et $\varepsilon^{\prime \prime}=f(T)$ à différentes fréquences pour AlN compacté.

Sur toutes les figures 2 à 8 , la fréquence est indiquée en $\mathrm{kHz}$; les courbes supérieures correspondent à $\varepsilon^{\prime \prime}$ et les courbes inférieures (tracées avec les mêmes traits) à $\varepsilon^{\prime}$. La température est indiquée en degrés Kelvin.

["Low temperature " curves $\varepsilon^{\prime}=f(T)$ and $\varepsilon^{\prime \prime}=f(T)$ at various frequencies for pressed AlN.

On the figures 2 to 8 , the frequency is indicated in $\mathrm{kHz}$; the upper curves concern $\varepsilon^{\prime \prime}$ and the lower curves (traces with the same dashes) with $\varepsilon^{\prime}$. The temperature is indicated in Kelvin.]

basse température de AlN compacté; on note la présence d'un pic d'absorption attribuable à l'adsorption de l'eau sur les grains : c'est un effet analogue à celui déjà observé [6] dans le cas de nombreux composés minéraux, tel que $\mathrm{Al}_{2} \mathrm{O}_{3}$ par exemple. Dans le cas de AlN fritté, nous avons vérifié expérimentalement que ce domaine d'absorption a naturellement disparu.

Les spectres diélectriques (courbes $\varepsilon^{\prime}=f(T)$ et $\varepsilon^{\prime \prime}=f(T)$ paramétrées en fréquence) haute température sont représentés sur les figures :

3 pour AlN compacté (échelle cinq fois plus petite que sur les figures suivantes)

4 pour AlN fritté sous charge

5 pour AlN fritté $+0,7 \% \mathrm{CaO}$ )

6 pour AlN fritté $+0,1 \% \mathrm{Cr}$ pourcentage

7 pour AlN fritté $+0,5 \% \mathrm{Cr}\}$ en moles.

8 pour AlN fritté $+2 \% \mathrm{Cr}$

La figure 9 représente les variations de $\varepsilon^{\prime \prime}$ en fonction de $\log v$, (paramétrées en température) pour AlN fritté et AlN + 0,1\% Cr.

Qualitativement, et dans un premier temps, on peut observer que le passage de AIN compacté à AIN fritté (Figs. 3 et 4) se traduit de deux manières : tout d'abord par une forte augmentation de $\varepsilon^{\prime}$ et des pertes diélectriques $\left(\varepsilon^{\prime \prime}\right)$, et aussi par l'apparition d'un pic surtout visible aux basses fréquences; ce pic a déjà été mis en évidence par d'autres auteurs [3] sans explications particulières.

Nous noterons tout de suite que l'introduction d'impureté $(0,7 \% \mathrm{CaO}$ ou de chrome métallique) fait disparaître ce pic d'absorption. L'intensité des pertes diélectriques dépend de la nature et de la concentration en impuretés introduites; cette propriété sera étudiée dans le paragraphe suivant à partir des courbes $\log \sigma=f(1 / T)$ (Figs. 10 et 11) choisies pour résumer les différents comportements; elles représentent les variations de la conductivité diélectrique $\sigma$ $\left(\sigma=\omega \varepsilon_{0} \varepsilon^{\prime \prime}\right)$ en fonction de l'inverse de la température. Nous pouvons voir que la conductivité, à température faiblement supérieure à l'ambiante, est de l'ordre de $10^{-10}-10^{-11} \Omega^{-1} \mathrm{~cm}^{-1}$ pour les échantillons frittés; ils se situent alors à la limite entre semi-conducteur et isolant qui est généralement fixée [7] à $\sigma \simeq 10^{-10} \Omega^{-1} \mathrm{~cm}^{-1}$. On remarquera également l'ordre de grandeur de la constante diélectrique (voisin de 9) de ces composés qui doivent donc être situés en (I) (constante diélectrique inférieure à 12) dans la classification proposée par Von Hippel [8]. 
$2 n " \varepsilon^{\prime \prime}$

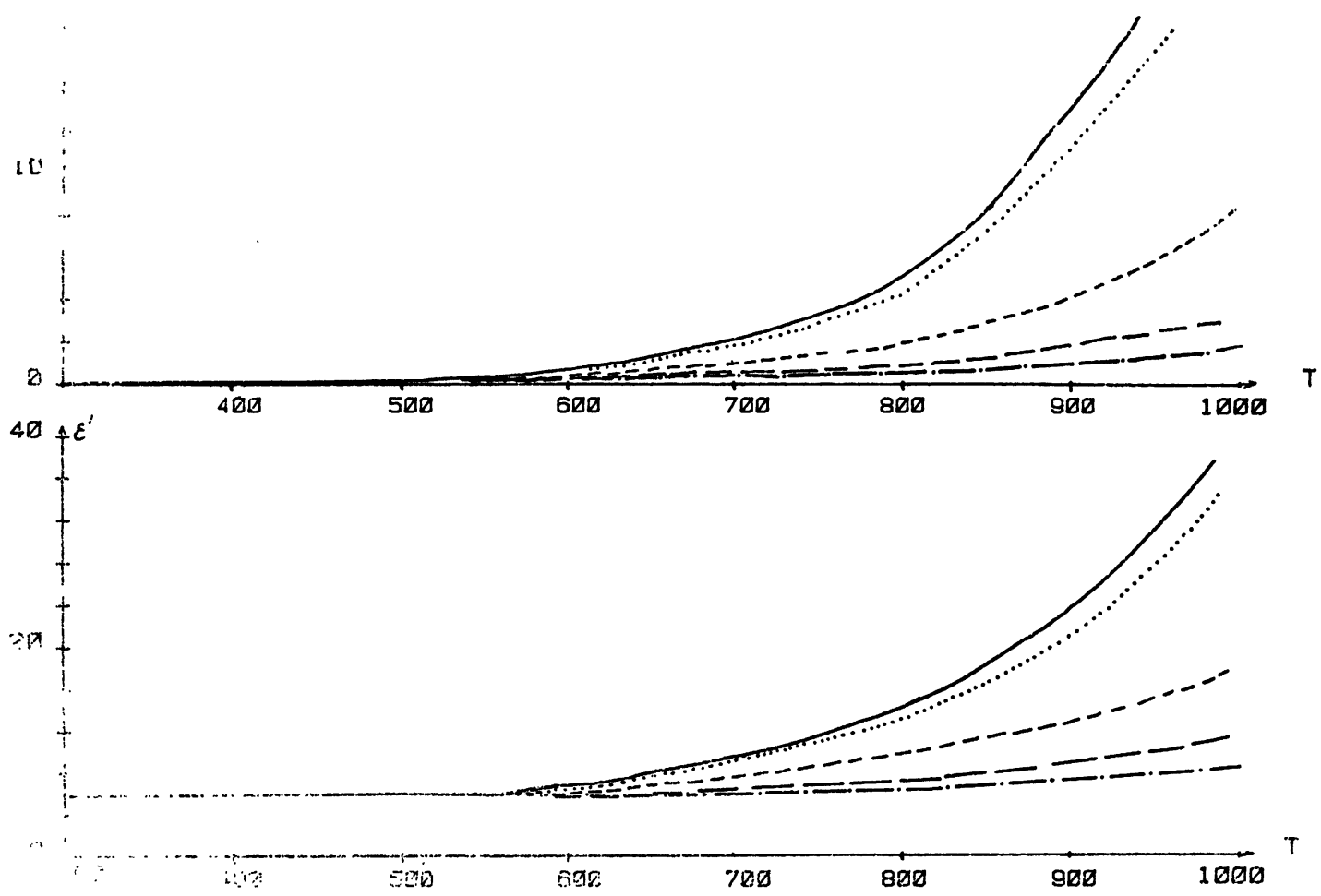

Fig. 3. - Courbes « haute température " $\varepsilon^{\prime}=f(T)$ et $\varepsilon^{\prime \prime}=f(T)$ à différentes fréquences pour AlN compacté. [" High temperature " curves $\varepsilon^{\prime}=f(T)$ and $\varepsilon^{\prime \prime}=f(T)$ at various frequencies for pressed AlN.]

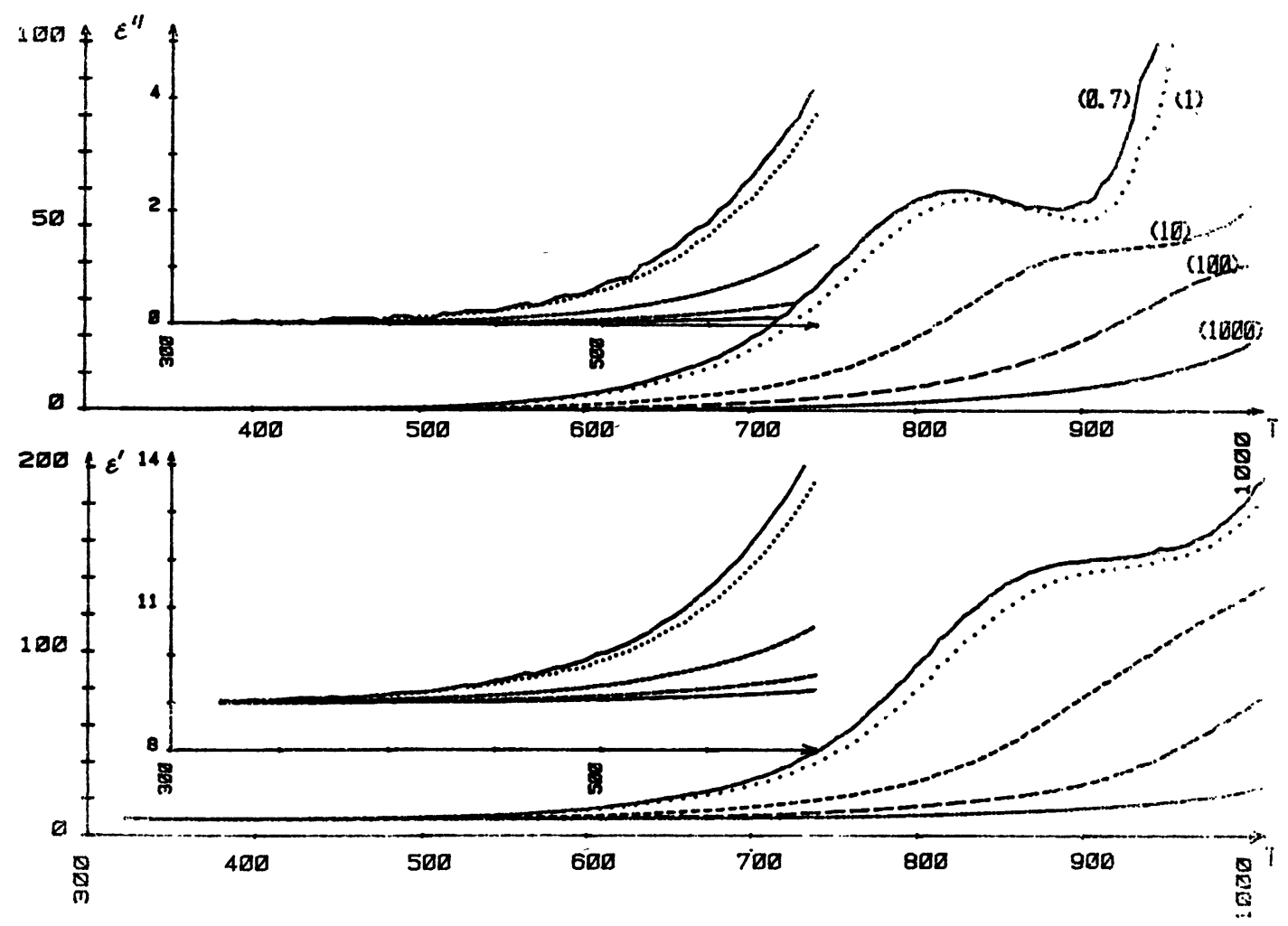

Fig. 4. - Courbes « haute température " $\varepsilon^{\prime}=f(T)$ et $\varepsilon^{\prime \prime}=f(T)$ à différentes fréquences pour AlN fritté.

[ " High temperature » curves $\varepsilon^{\prime}=f(T)$ and $\varepsilon^{\prime \prime}=f(T)$ at various frequencies for hot pressed Aln.] 
4. Interprétation des résultats et discussions. 4.1 INTERPRÉTATION DE LA PRÉSENCE D'UN PIC D'ABSORPTION DANS AIN FRITTÉ. - On a vu (Figs. 3 et 4) que le passage de AlN pressé à AlN fritté se traduit par l'apparition d'un pic d'absorption particulièrement visible aux plus basses fréquences. Le passage de l'échantillon pressé à l'échantillon fritté se traduit physiquement par une très forte diminution de la porosité $(45 \%$ à pratiquement $0 \%$ ) mais aussi par "l'introduction " de l'oxynitrure d'aluminium ciaprès désigné par AlON (absent dans le produit pressé) dans le produit fritté [9]. La variation de la porosité (et des défauts physiques de réseau en résultant) ne peut pas être tenue comme responsable de l'apparition du pic, car ce dernier n'existe pas dans un échantillon fritté de $\mathrm{AlN}+0,7 \% \mathrm{CaO}$ (Fig. 5).

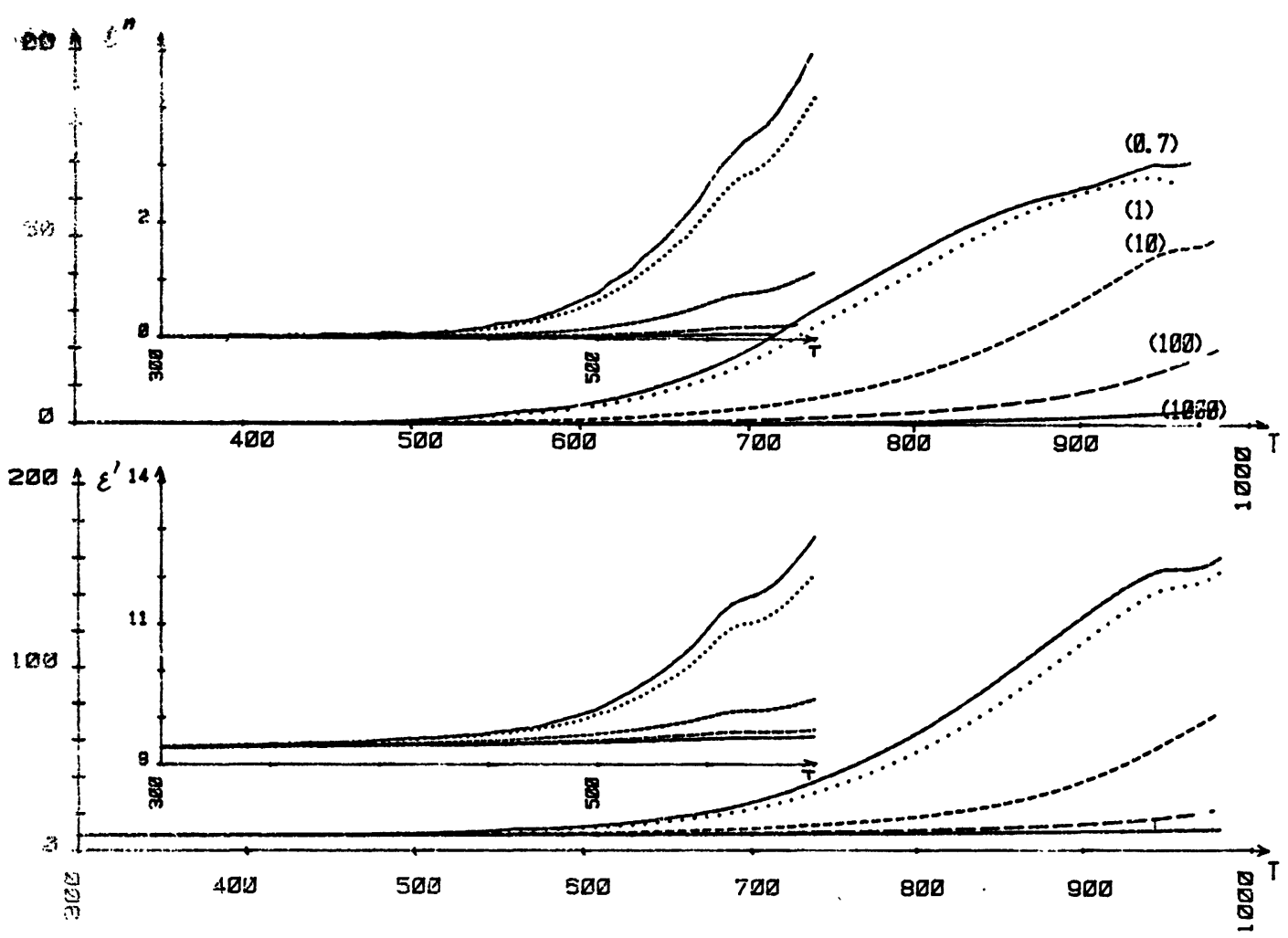

Fig. 5. - Courbes « haute température " $\varepsilon^{\prime}=f(T)$ et $\varepsilon^{\prime \prime}=f(T)$ à différentes fréquences pour AlN fritté $+0,7 \% \mathrm{CaO}$.

[" High temperature " curves $\varepsilon^{\prime}=f(T)$ and $\varepsilon^{\prime \prime}=f(T)$ at various frequencies for hot pressed $\mathrm{AlN}+0.7 \% \mathrm{CaO}$.]

Par contre il a été montré [9] que, dans ce dernier échantillon, il n'existait pas de composé AlON, qui doit être ainsi rendu responsable du phénomène étudié. Nous avons par ailleurs vérifié aussi par diffraction $X$ que AlON n'existait pratiquement pas dans les échantillons frittés $\mathrm{AlN}+\mathrm{Cr}$, et pour lésquels le pic d'absorption étudié n'existe pas non plus (Figs. 6, 7, 8).

Nous pouvons donc conclure que le pic d'absorption diélectrique observé dans le cas de AlN fritté est dû à un défaut de réseau du type [10] «imperfection chimique ": AlON, qui introduit une polarisabilité interfaciale. Le comportement diélectrique d'un tel système peut être décrit par le modèle à couche du type Maxwell-Wagner; le terme dipolaire contenu alors dans l'expression théorique de $\varepsilon^{\prime \prime}$ explique ainsi la présence et la forme du pic d'absorption étudié.

Ce phénomène est également visualisé sur les courbes plus classiques (Fig. 9) $\varepsilon^{\prime \prime}=f(\log v)$ relatives a AlN fritté. Comme on pouvait s'y attendre [12], le pic dû à l'effet M.W. a tendance à se produire à des fréquences inférieures à $1 \mathrm{kHz}$; on atteint ainsi la limite inférieure de fonctionnement de notre spectromètre et des mesures à plus basses fréquences seraient nécessaires pour une étude plus détaillée. On peut toutefois remarquer qu'une étude " théorique " serait illusoire dans notre cas; en effet il nous faudrait connaître à la fois les valeurs de $\varepsilon^{*}$ de AlON (composé que l'on n'a pas obtenu pur) et surtout l'ordre de grandeur de l'épaisseur des couches de AlON ainsi que leur nombre. Sans ces données (pratiquement impossibles à obtenir dans notre cas), toute tentative de calcul ne pourrait conduire qu'à des résultats peu crédibles.

Par ailleurs, sur cette même figure 9, on peut remarquer que pour $\mathrm{AlN}+0,1 \% \mathrm{Cr}$ (il en va de même pour les autres composés contenant des ajouts et $\mathrm{AlN}$ compacté) la variation de $\varepsilon^{\prime \prime}$ en fonction de la fréquence ne présente pas de pic (pas d'effet M.W. comme on l'a remarqué précédemment sur les figures 


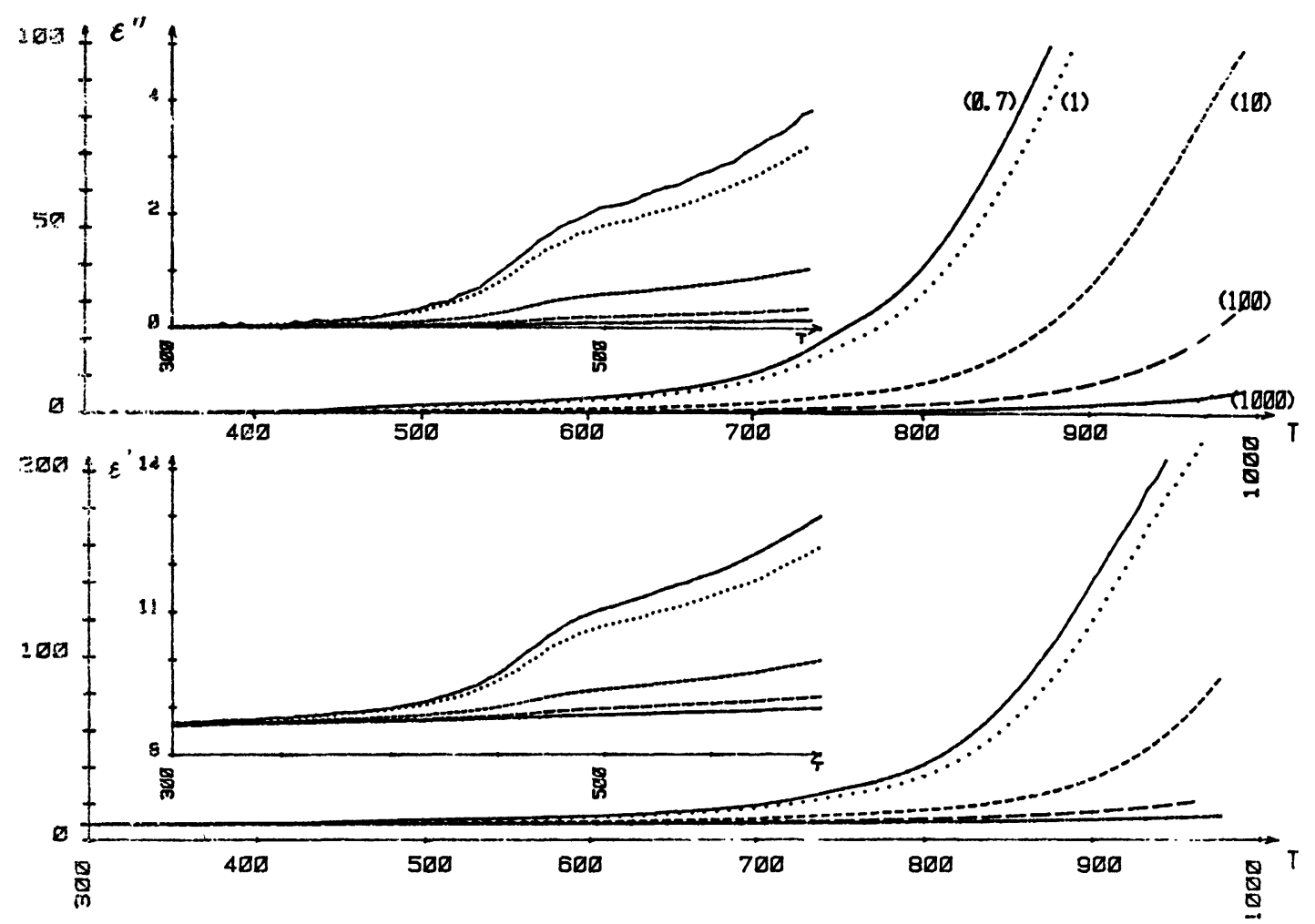

Fig. 6. - Courbes « haute température » $\varepsilon^{\prime}=f(T)$ et $\varepsilon^{\prime \prime}=f(T)$ à différenteș fréquences pour AlN fritté $+0,1 \% \mathrm{Cr}$. [" High temperature " curves $\varepsilon^{\prime}=f(T)$ and $\varepsilon^{\prime \prime}=f(T)$ at various frequencies for hot pressed AlN $+0.1 \% \mathrm{Cr}$.]

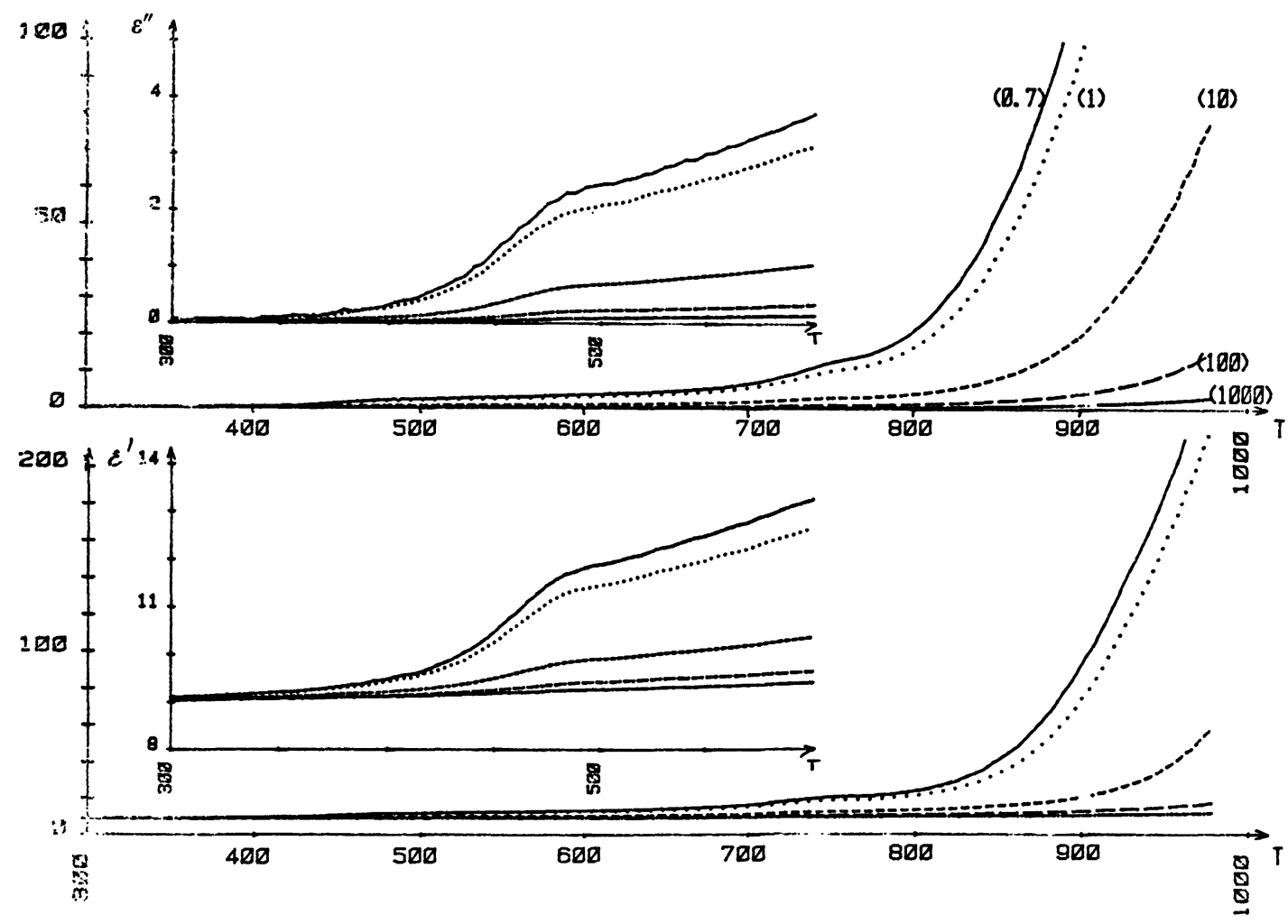

Fig. 7. - Courbes " haute température " $\varepsilon^{\prime}=f(T)$ et $\varepsilon^{\prime \prime}=f(T)$ à différentes fréquences pour AlN fritté $+0,5 \%$ Cr. [" High temperature " curves $\varepsilon^{\prime}=f(T)$ and $\varepsilon^{\prime \prime}=f(T)$ at various frequencies for hot pressed AlN $+0.5 \% \mathrm{Cr}$.] 


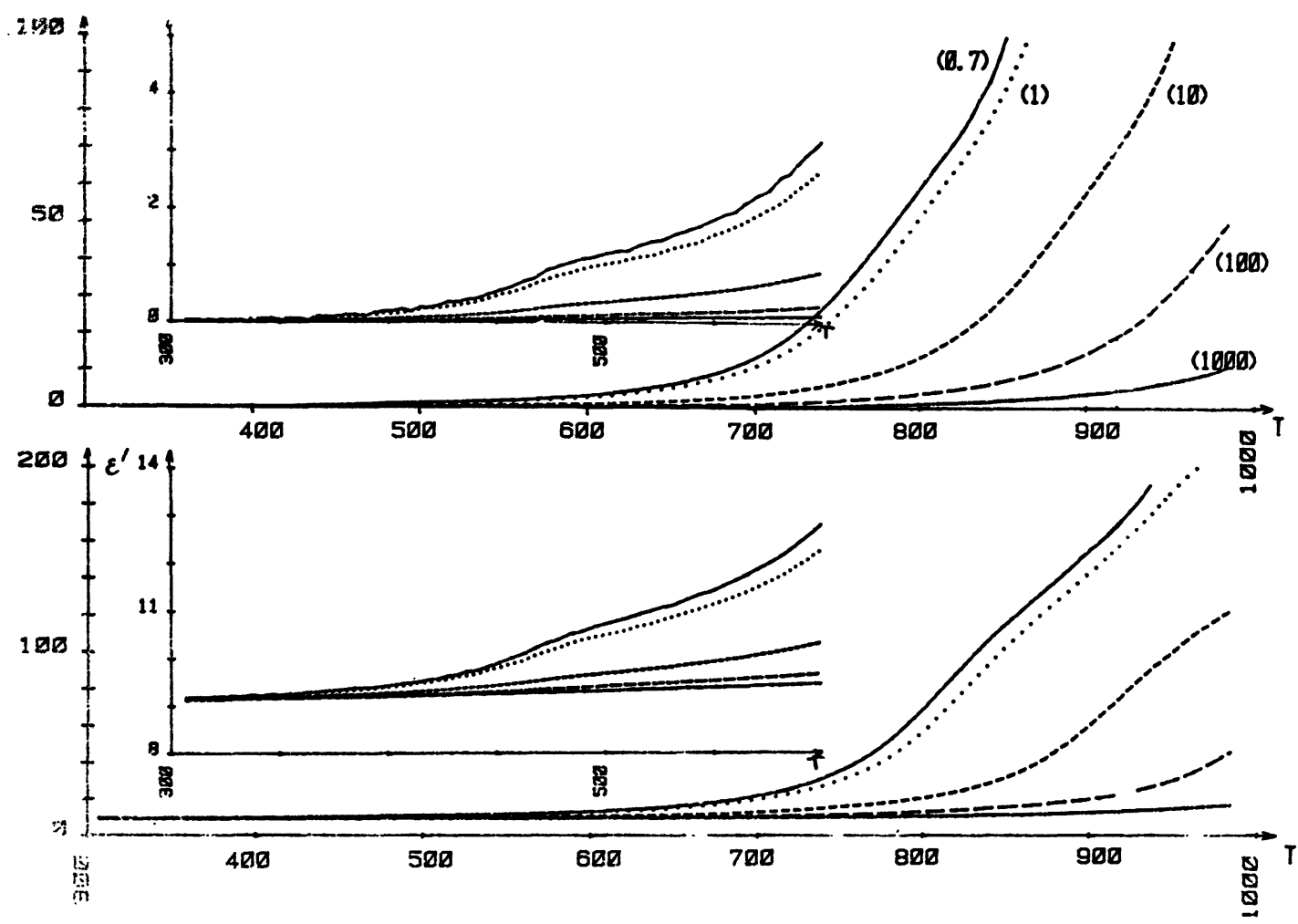

Fig. 8. - Courbes « haute température » $\varepsilon^{\prime}=f(T)$ et $\varepsilon^{\prime \prime}=f(T)$ à différentes fréquences pour AlN fritté $+2 \% \mathrm{Cr}$. [" High temperature " curves $\varepsilon^{\prime}=f(T)$ and $\varepsilon^{\prime \prime}=f(T)$ at various frequencies for hot pressed AlN $+2 \% \mathrm{Cr}$.]

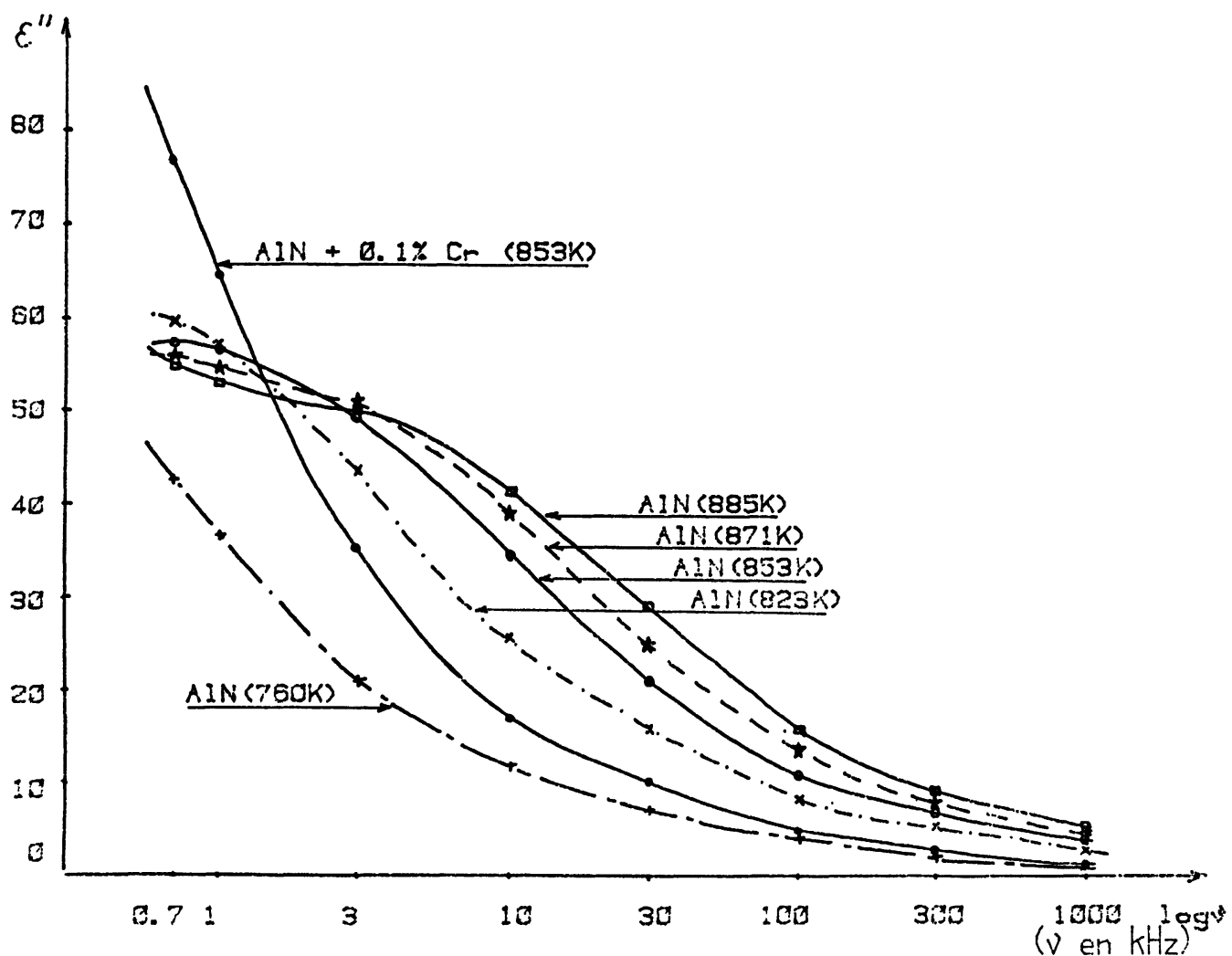

Fig. 9. - Courbes $\varepsilon^{\prime \prime}=f(\log v)$ à différentes températures pour $\mathrm{AlN}$ fritté et $\mathrm{AlN}+0,1 \% \mathrm{Cr}$.

[Curves $\varepsilon^{\prime \prime}=f(\log v)$ at various temperatures for hot pressed $\mathrm{AlN}$ and $\mathrm{AlN}+0.1 \% \mathrm{Cr}$.] 
tracées en fonction de la température) et est du type

$$
\varepsilon^{\prime \prime}=\frac{\sigma}{2 \pi v \varepsilon_{0}} \simeq f\left(\frac{1}{v}\right)
$$

c'est un effet de conductivité qui croît aux plus basses fréquences [12] et dû aux charges du réseau.

4.2 ORIGINE DES PERTES DiÉLECTRIQUeS ET DE LA CONDUCTIVITÉ. - On a noté expérimentalement l'existence de pertes diélectriques dont l'importance croît avec la température, notamment aux plus basses fréquences. Le passage de AlN compacté à AlN fritté se traduit par une forte augmentation de ces pertes diélectriques qui ne peut pas être attribuée à $\mathrm{AlON}$; en effet, ce fort accroissement se produit également en passant de $\mathrm{AlN}$ compacté à AlN $+0,7 \% \mathrm{CaO}$ fritté qui ne contient pas de AlON; cette même remarque peut aussi être faite pour les échantillons frittés AlN + Cr. Par ailleurs [11] il a déjà été noté pour d'autres cas, qu'une diminution de la porosité augmentait $\varepsilon^{\prime}$ et $\varepsilon^{\prime \prime}$. De plus, et de façon générale, on peut dire que l'origine des pertes diélectriques ne peut pas être :

- due à des charges libres dans le réseau car la conductivité devrait être indépendante de la fréquence [12], ce qui n'est pas le cas ;

- de nature dipolaire à cause de l'absence en général de pic d'absorption (exception faite de AlN fritté pour lequel il existe, comme nous l'avons vu, en plus un "défaut" de nature chimique).

Compte tenu des fréquences auxquelles est effectuée l'étude, la seule origine qui reste possible pour ces pertes diélectriques est celle due à l'existence de charges superficielles liées à des défauts de réseau du type "imperfection physique " [10] (dislocations...). (Une hypothèse formulée dans ce même sens a été faite pour des cas similaires [13]). L'effet qui en résulte est du type Maxwell-Wagner-Sillars, modifié par la présence des barrières de potentiel au contact diélectrique, résultant de la présence de charges dans les états de surface [14]. La condition de continuité de la composante normale de l'induction électrique au contact interdiélectrique n'est plus valable compte tenu de la présence des charges; il en résulte une modification du schéma équivalent et de l'expression analytique (par rapport à la théorie de Maxwell-Wagner) des grandeurs $\varepsilon^{\prime}$ et $\varepsilon^{\prime \prime}$ en fonction de la fréquence. La complexité du problème doit alors faire intervenir plusieurs phénomènes dont il est difficile de connaitre ici l'importance relative : accumulation des porteurs dans les barrières de potentiel, effet de la nature exacte des états de surface et importance des pièges.

Compte tenu de toutes ces inconnues, nous ne proposerons pas de schéma théorique qui pourrait alors être discutable; nous signalerons simplement qu'un calcul théorique effectué par Goffaux [14] dans le cas de "l'équilibre dynamique d'une assemblée de porteurs libres accumulés dans une barrière de potentiel pour un champ électrique périodique et redistribués dans le volume du diélectrique par un mécanisme de diffusion ", conduit pour la conductance et donc la conductivité, à un terme qui croît avec la fréquence alors que le terme capacitif, c'est-à-dire en fait $\varepsilon^{\prime}$, diminue lorsque la fréquence augmente : c'est ce que

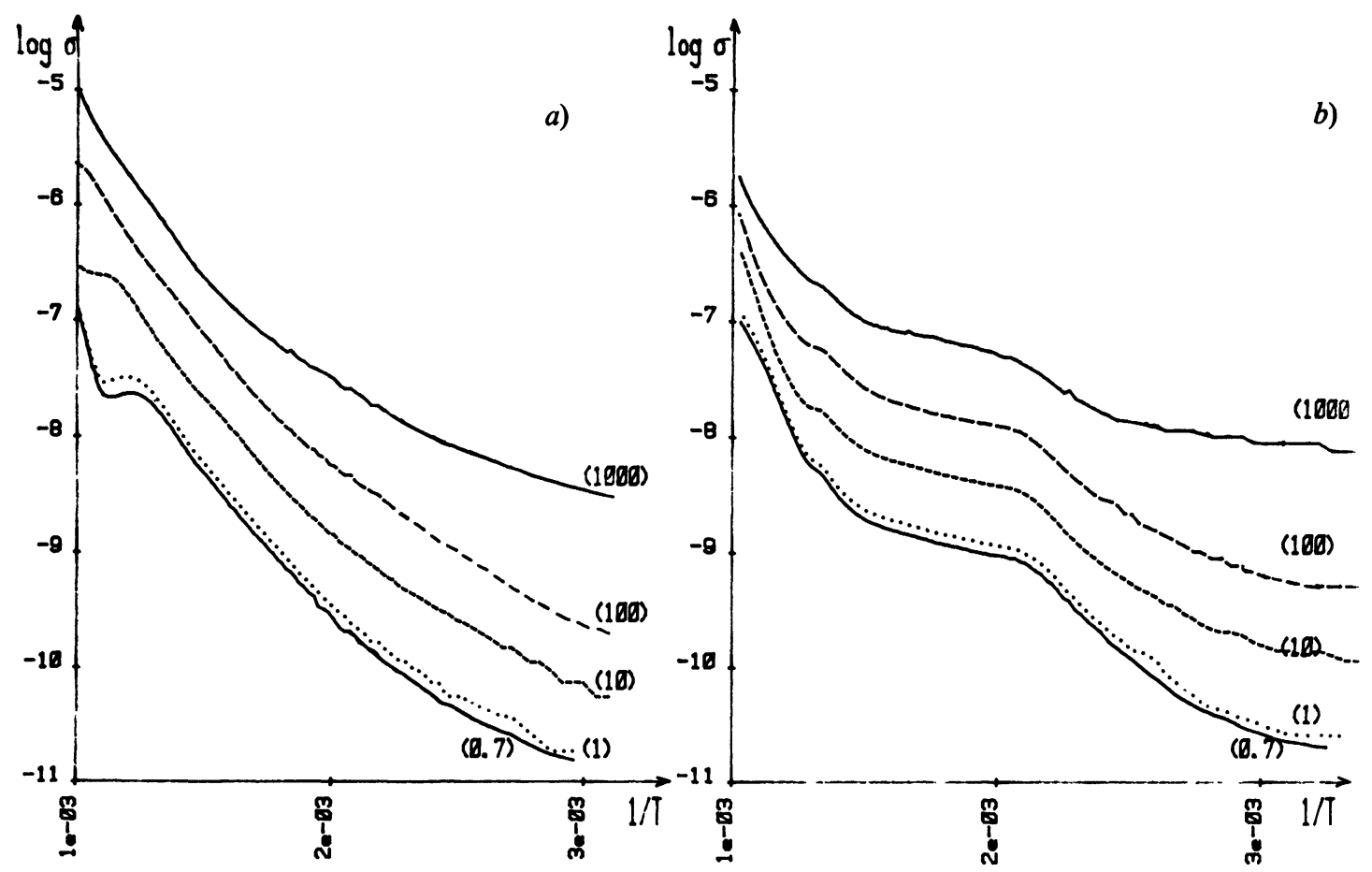

Fig. 10. - Courbes $\log \sigma=f(1 / T)$ à différentes fréquences pour $(a):$ AlN fritté ; $(b):$ AlN fritté $+0,5 \%$ Cr.

[Curves $\log \sigma=f(1 / T)$ at various frequencies for $(a)$ : hot pressed $\mathrm{AlN} ;(b):$ hot pressed $\mathrm{AlN}+0.5 \% \mathrm{Cr}$.] 
l'on observe effectivement expérimentalement pour $\sigma$ et $\varepsilon^{\prime}$ (Figs. 10 et 3 à 8 ).

L'augmentation des pertes diélectriques entre le produit compacté et les produits frittés peut alors être expliquée par l'accroissement du nombre des grains qui peuvent présenter ces imperfections physiques génératrices des pertes diélectriques en relations avec les différents facteurs mentionnés. L'adjonction du chrome métallique (déjà utilisé comme " dopant » dans le cas de $\mathrm{Al}_{2} \mathrm{O}_{3}$ et dont le rayon ionique est voisin de celui de $\mathrm{Al}$ ) augmente les pertes diélectriques et la conductivité pour des températures comprises entre l'ambiante et $650 \mathrm{~K}$ : l'introduction d'une telle impureté (dont l'effet maximum est obtenu pour une concentration de l'ordre de $0,5 \%$ et relativement faible, comme c'est souvent le cas [10]), augmente les charges d'espace ainsi que les défauts physiques de réseau auxquels elles sont liées. On doit en outre remarquer que dans cette zone de température, la pente de

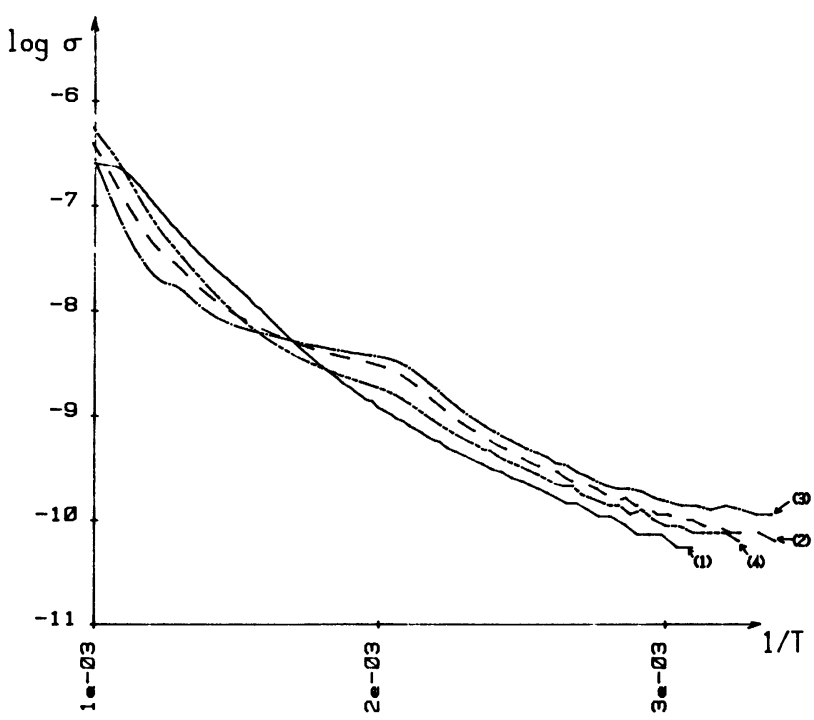

Fig. 11. - Courbes comparatives $\log \sigma=f(1 / T)$ à $10 \mathrm{kHz}$ pour les différents échantillons étudiés : (1) : AlN fritté ; (2) AlN fritté $+0,1 \% \mathrm{Cr}$; (3) : AlN fritté $+0,5 \% \mathrm{Cr}$; (4) : AlN fritté $+2 \% \mathrm{Cr}$.

[Comparative curves $\log \sigma=f(1 / T)$ at $10 \mathrm{kHz}$ for the different samples studied : (1) hot pressed AlN; (2) hot pressed $\mathrm{AlN}+0.1 \% \mathrm{Cr}$; (3) hot pressed AlN $+0.5 \% \mathrm{Cr}$; (4) hot pressed $\mathrm{AlN}+2 \% \mathrm{Cr}$.] $\log \sigma=f(1 / T)$ est pratiquement la même pour tous les corps : l'énergie d'activation (sensiblement indépendante de la fréquence et voisine de $0,08 \mathrm{eV}$ et suffisamment faible pour penser que les charges sont de nature électronique et non ionique) semble pratiquement indépendante de la présence et de la concentration en chrome (contrairement au cas de $\mathrm{Al}_{2} \mathrm{O}_{3}$ dopé au chrome [13]) : le "dépiégeage " des charges dans les défauts de réseau nécessite la même énergie; elle ne peut dépendre que de la nature du défaut qui semble être ainsi la même dans tous les cas; seul, ainsi qu'on l'a déjà supposé, le nombre de ces charges liées aux défauts semble être augmenté lors du dopage par le chrome métallique. A plus haute température, et pour le cas des échantillons dopés au chrome, le changement de courbure de $\log \sigma=f(1 / T)$ peut être rattaché à celui qui se produit dans le cas des semiconducteurs extrinsèques (toute proportion gardée, la nature de la conduction étant différente) : il pourrait là aussi se produire, à partir d'une certaine température, une saturation de la conductivité générée par la présence du chrome. Une augmentation plus importante de la température pourrait à nouveau stimuler la conductivité dont la nature serait quelque peu différente : dans tous les cas, la pente de $\log \sigma=f(1 / T)$ augmente et correspond ainsi aux plus hautes températures à un phénomène de conduction qui nécessite des énergies d'activation plus élevées.

5. Conclusion. - Nous avons montré l'existence d'une double origine pour les pertes diélectriques présentées à haute température par AIN fritté : l'une due à des imperfections chimiques (AlON), l'autre causée par des charges liées physiquement à des défauts de réseau. Il en résulte finalement que AIN présente une conductivité très légèrement inférieure aux semiconducteurs classiques (mais dont la nature de conduction est bien différente). A hautes températures les pertes diélectriques ne sont relativement faibles qu'à des fréquences assez élevées $\left(10^{5} \mathrm{~Hz}\right.$ ou $\left.10^{6} \mathrm{~Hz}\right)$, ce qui peut limiter l'utilisation de AIN en tant qu'isolant. A température ambiante, les pertes diélectriques $\left(\varepsilon^{\prime \prime} \simeq 10^{-3}\right)$ relativement faibles permettent cependant de considérer AlN comme un assez bon diélectrique.

\section{Bibliographie}

[1] Boch, P., Glandus, J. C., Jarrige, J., Lecompte, J. P., Mexmain, J., Sintering, oxidation and mechanical properties of hot pressed aluminium nitride. Ceramurgia International 11 (1981) 3093.

[2] Komeya, K., Noda, F., Toshiba Rev. 92 (1974) 13.

[3] Taylor, K. H., Camille Lenie, Some properties of aluminium nitride. J. Electrochem Soc. 4 (1960) 308.
[4] Anbreeva, T. U., Dubovick, T. V., Aluminium nitride is a high temperature dielectric. Dieletriki $I$ Poluprovodniki 6 (1974) 31.

[5] Moliton, A., Decossas, F., Moliton, J. P., Revue Phys. Appl. 15 (1980) 1563.

[6] Daniel, V., Dielectric relaxation (Academic Press) 1967. 
[7] Wilson, A. H., The theory of metals (Cambridge Uni- [11] KInGERY, W. D., Introduction to ceramics (John Wiley) versity Press) 1965.

[8] Von Hippel, A. R., Les diélectriques et leurs applications (Dunod), 1961.

[9] SAKAI, T., Yogyo Kyokai Ski, 86 (1978) 125.

[10] FreymanN, R., Soutif, M., La spectroscopie hertzienne (Dunod) 1960. 